\title{
Colgajos de las perforantes del pie: problemas y posibles soluciones
}

\author{
Foot perforator flaps: \\ problems and possible solutions
}

\author{
Prousskaia Peregudova, E.*, Gabilondo Zubizarreta, F. J .**, Salvador Sanz, J . F.***
}

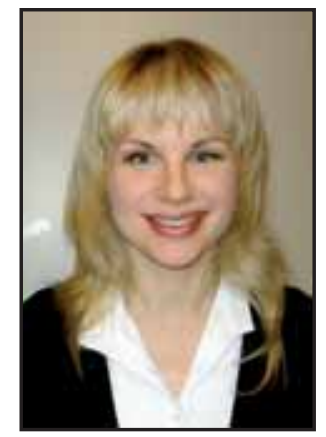

Prousskaia Peregudova, E.

\section{Resumen}

A pesar de diversas publicaciones existentes sobre el colgajo mediano plantar tradicional, los colgajos de las perforantes del pie no están suficientemente estudiados. Es necesario ampliar nuestros conocimientos sobre la anatomía vascular de esta región, los problemas que pueden surgir durante la disección de la zona y las posibles soluciones.

Realizamos disección anatómica de 10 pies de cadáver utilizando relleno vascular con látex coloreado. Definimos la secuencia de la disección de las perforantes de la arteria plantar medial. Medimos los puntos de salida de las perforantes dominantes en relación con 3 puntos de referencia, diámetro y longitud de cada perforante. Evaluamos la utilidad de cada colgajo para su uso en reconstrucción local o a distancia en función del diámetro y la longitud de la perforante, grosor del colgajo y morbilidad de la zona donante.

Los colgajos de los vasos perforantes del pie son una buena opción para la reconstrucción tanto local como a distancia, con una mínima morbilidad de la zona donante y con un buen resultado funcional gracias a las características del tejido del pie cuando se usa para reconstrucción en mano.

$\begin{aligned} \text { Palabras clave } & \text { Perforantes plantares, Colgajos planta- } \\ & \text { res, Arteria plantar media. } \\ \text { Código numérico } & 158336\end{aligned}$

Traditional medial plantar flap is widely described in the literature, but foot perforator flap is a relatively new subject which still needs more detailed anatomical investigation. We need to amplify our knowledge about this surgically challenging region, dissection difficulties and possible solutions.

We performed anatomical dissection on 10 cadaver feet with dyed latex intravascular injection and then dissected out the perforators arising from the medial plantar artery. We measured the origin of the dominant perforators in relationship to 3 reference points, diameter and length of each perforator. We analyzed the application of flaps based on these perforators as local and free flaps in relation to pedicle length and diameter, flap thickness and donor site morbidity.

Foot perforator flaps are a good option for local and distant reconstruction. Donor site morbidity is low and functional result is good due to tissue characteristics of the foot region when used for hand reconstruction.

* Especialista en Cirugía Plástica, Profesor Titular del Departamento de Anatomía e Histología Humanas de la Universidad Miguel Hernández de Elche, Alicante (España). 


\section{Introducción}

Al ser una zona de carga, el pie exige su reconstrucción con tejidos que proporcionen una buena resistencia, por lo que, el cierre con el injerto de piel en las zonas de apoyo, resulta inviable en la mayoría de los casos. Los colgajos locales pediculados, al rotarlos provocan un gran abultamiento, comprometiendo el resultado final de la reconstrucción. Sin embargo, la rotación de un colgajo basado solo en el pedículo vascular proporciona mejor resultado tanto estético como funcional para cubrir unas zonas tan importantes a la hora de caminar como son el talón o el tendón de Aquiles. Además de las pérdidas de sustancia en el pie, a menudo nos enfrentamos con la necesidad de reconstruir otra parte de cuerpo humano tanto o más importante como es la mano. Los tejidos de la zona palmar tienen mucho en común con los tejidos de la zona plantar y el tamaño de los vasos del colgajo coincide con el diámetro de los vasos digitales, hecho que nos permite una magnífica posibilidad de reconstrucción con tejido prácticamente idéntico para la palma de la mano y la superficia flexora de los dedos.

Gracias a todo esto, el colgajo de la zona plantar está considerado como una de las mejores opciones para reconstrucción del pie y de la mano. En nuestro trabajo presentamos un estudio anatómico sobre las perforantes de arteria plantar medial; disecamos las perforantes dominantes y definimos los colgajos correspondientes a las perforantes seleccionadas. Analizamos la posibilidad de usar cada colgajo, tanto para reconstrucción local como para reconstrucción a distancia, dependiendo del diámetro y longitud de los pedículos de cada colgajo.

\section{Bases anatómicas:}

La planta del pie está constituida por cuatro capas: piel, grasa subcutánea, fascia y musculatura plantar. La piel del pie tiene un grosor suficiente para aguantar la carga continua del peso corporal. Las glándulas sebáceas y el pelo existen solo en el dorso del pie.

La grasa subcutánea es escasa en el dorso del pie y abundante en la planta, donde además está dividida por tractos fibrosos que comunican la dermis y la fascia plantar.

La fascia plantar es un tejido conectivo muy fuerte que se inicia en el calcáneo y se inserta en las cabezas de los cinco metatarsianos. Numerosos septos de la fascia dividen la planta del pie en 3 compartimentos: el de la musculatura que se inserta en el quinto dedo es el compartimiento lateral, el de la musculatura que se inserta en la base del primer dedo es el comparti- miento medial y el de la musculatura que se inserta en el segundo, tercer y el cuarto dedos es el compartimiento medio.

La musculatura del pie está dividida en: musculatura dorsal, con el extensor digitorum brevis lateral y el extensor hallucis medial y musculatura plantar del pie que contiene 4 capas musculares:

- Capa superficial: formada por el abductor hallucis, el abductor digiti minimi y el flexor digitorum brevis.

- Segunda capa; contiene el flexor accesorius y los lumbricales.

- Tercera capa: en la que están el flexor hallucis brevis, flexor digiti minimi brevis, adductor hallucis obliquus y adductor hallucis transversus.

- Cuarta capa: contiene la musculatura interósea.

La base ósea del pie está representada por 3 grupos de huesos:

- Primer grupo: formado por el os calcaneus, el os talus, el os cuboideum, el os naviculare y el os cuneiforme.

- Segundo grupo: formado por el metatarso con sus 5 huesos.

- Tercer grupo: compuesto por los 14 huesos de las falanges digitales.

El pie está vascularizado por 3 troncos vasculares arteriales: a. peroneus, a. tibialis anterior que continúa como la a. dorsalis pedis y a. tibialis posterior que se bifurca en a. plantaris medial y a. plantaris lateral. El retorno venoso se realiza por la vena saphena magna y por la vena saphena parva. Además cada arteria habitualmente está acompañada de 2 venas.

La inervación de planta del pie se realiza por el nervio tibial posterior que se bifurca en n. plantaris lateral y $\mathrm{n}$. plantaris medial. El dorso del pie está inervado por $n$. peroneus y n. saphenus (1-3).

\section{Material y método}

Realizamos un estudio sobre 10 preparados de pie de cadáver con relleno vascular de látex coloreado en el Departamento de Anatomía e Histología Humanas de la Universidad Miguel Hernández de Elche, Alicante (España).

Nos proponemos como objetivos científicos del estudio:

1. Establecer un modelo de disección experimental de las perforantes de la arteria plantar medial en cadáver.

2. Definir las perforantes dominantes de esta arteria y describir los colgajos nutridos por estas perforantes.

3. Evaluar la utilidad de cada colgajo para reconstrucción local o a distancia en función del diámetro y de la longitud de la perforante, grosor del colgajo y morbilidad de la zona donante. 


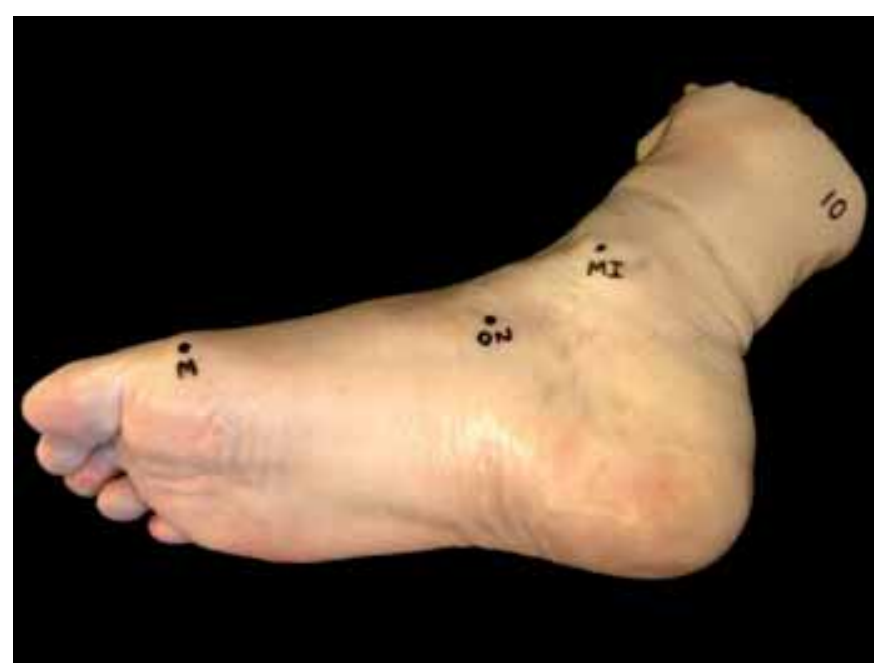

Fig. 1. Tres puntos de referencia para la medición de la salida de las perforantes.

\section{Técnica quirúrgica}

Para la medición de los puntos de salida de las perforantes marcamos 3 puntos de referencia (Fig. 1):

Punto 1 - Maleolo interno.

Punto 2 - Hueso navicular.

Punto 3 - Primera articulación metatarsofalángica.

La disección comienza en el borde superior del retináculo siguiendo el trayecto de la arteria tibial posterior. Se disecan todas las perforantes. Las perforantes que penetran en el retináculo tienen un patrón inconstante y el diámetro es menor de 0,5 mm. Observamos una media de 3-4 perforantes que salen de la arteria tibial posterior a su paso por detrás de retináculo (Fig. 2), pero la variabilidad anatómica y el pequeño diámetro de estas perforantes no proporcionan una base segura para la disección de un colgajo.

Al pasar el retináculo nos encontramos con el espacio entre el retináculo y el músculo abductor hallucis, donde en todas nuestras disecciones observamos una perforante grande que sale del tronco principal de la a. mediana plantar nutriendo la piel que corresponde a la

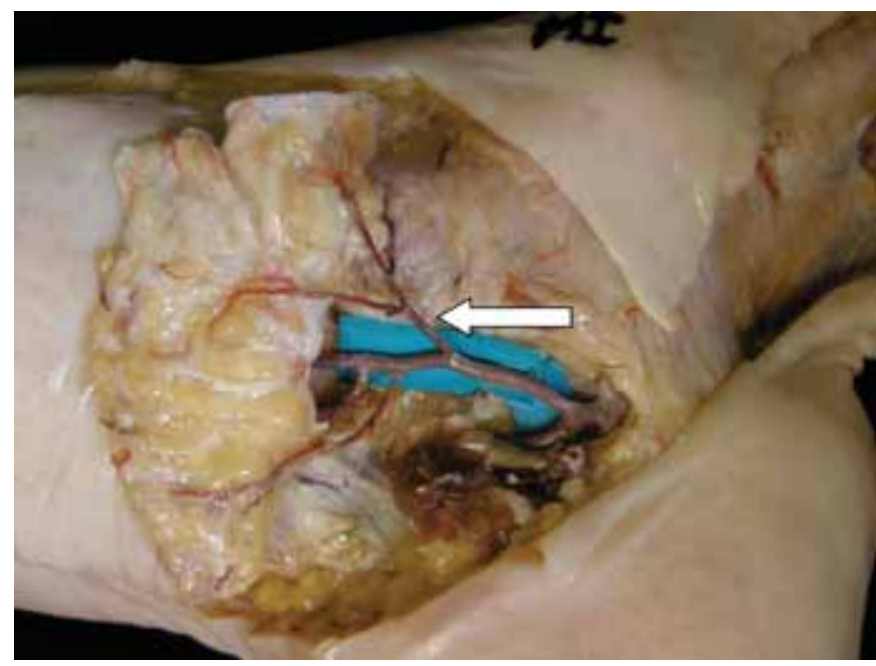

Fig. 3. Perforante dominante de la zona I.

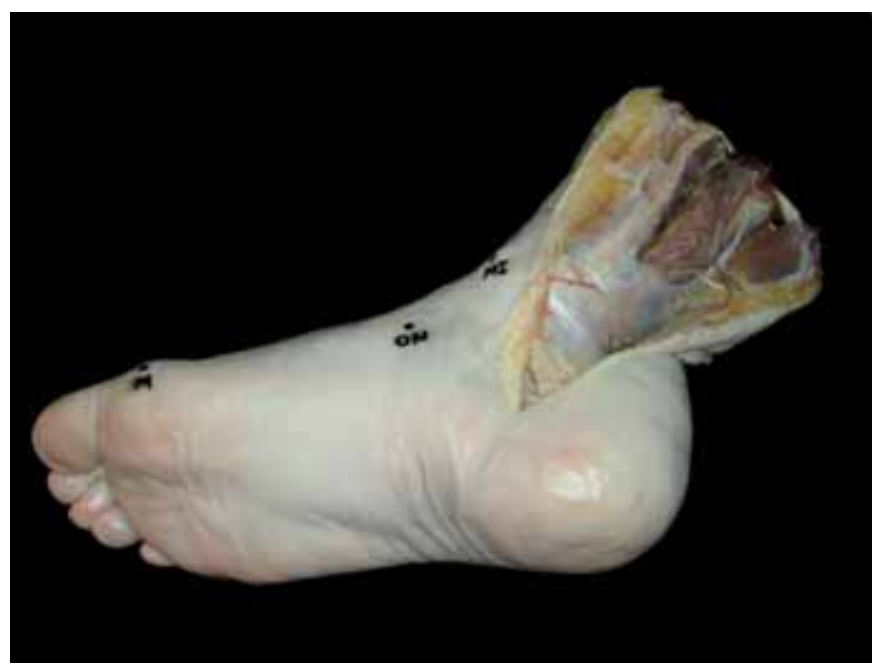

Fig. 2. Perforantes que penetran el retináculo.

zona entre el maleolo interno y el hueso navicular (Fig. 3).

La piel de esta zona tiene un grosor muy fino y gracias a su elasticidad permite un cierre directo, tomando un colgajo de $2 \mathrm{~cm}$ de anchura. La zona nutrida por esta perforante se nombra como zona I. Una vez disecada la perforante, las mediciones se toman desde el punto de salida de la perforante hasta cada uno de los 3 puntos de referencia mencionados anteriormente.

Después de levantar el colgajo de la zona I con su perforante, disecamos la perforante desde su inicio en el tronco principal de la arteria mediana plantar hasta su entrada en la piel y tomamos mediciones de su longitud y diámetro con una escala de $0,5 \mathrm{~mm}$. El diámetro medio de la perforante según nuestras mediciones es de 1,14 mm y varía de 0,8 a $1,1 \mathrm{~mm}$; la longitud media es de 1,5 $\mathrm{cm}$ siendo la longitud mínima de 1,1 y la máxima de 2,6 $\mathrm{cm}$, sin disecar el tronco principal de la arteria mediana plantar.

La segunda perforante dominante sale por debajo del músculo abductor hallucis nutriendo la zona del

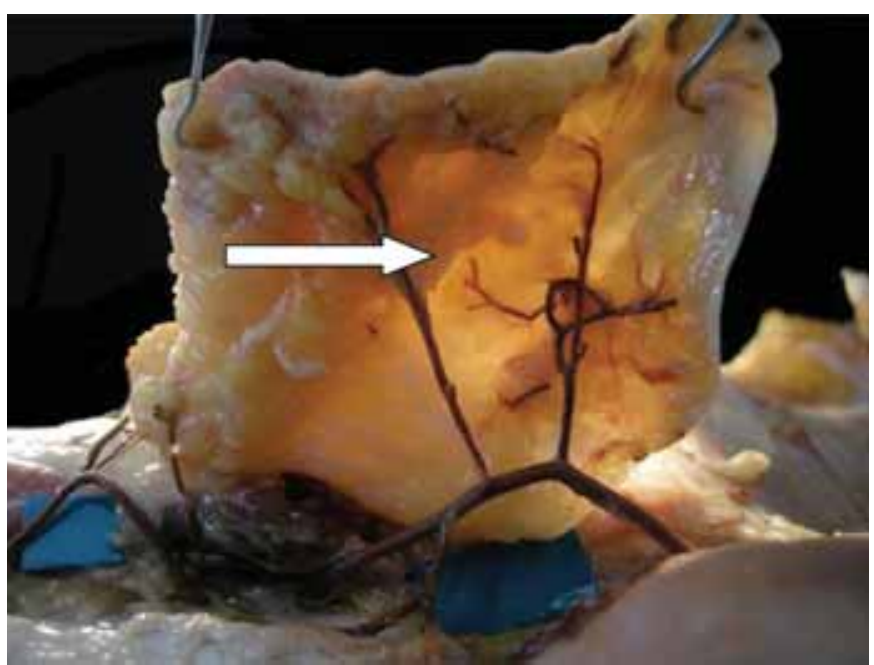

Fig. 4. Perforante dominante de la zona II. 


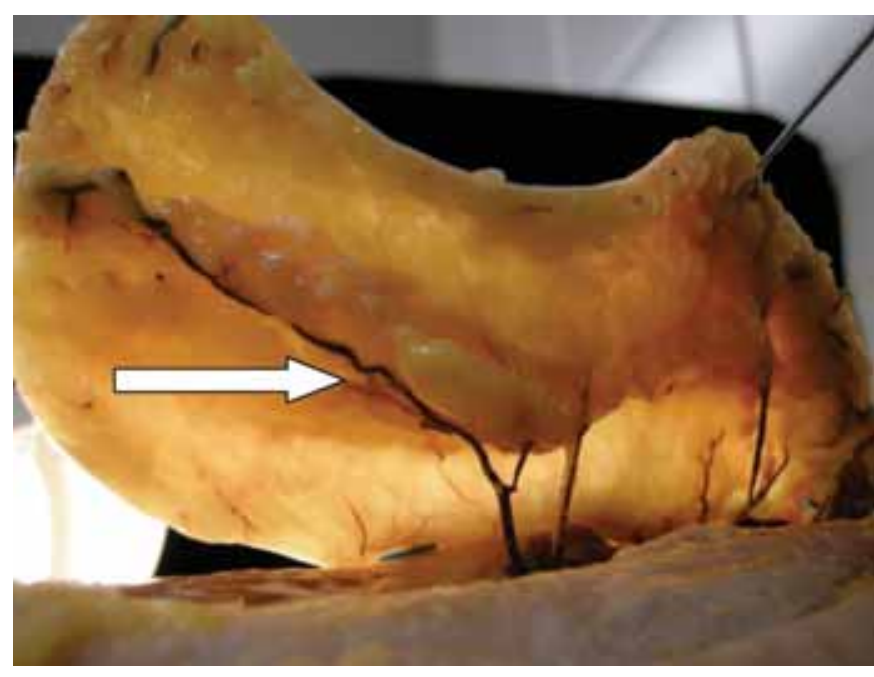

Fig. 5. Nutrición del colgajo medialis pedis. Zona III.

colgajo mediano plantar tradicional (Fig. 4). Denominamos a esta zona, zona II. Se toman las mismas mediciones que en la zona I. El diámetro medio de esta perforante, según nuestras mediciones es 0,89 mm y varía de 0,7 a $1,1 \mathrm{~mm}$; la longitud media es 1,99 $\mathrm{cm}$ con un rango de 1,3 a $3 \mathrm{~cm}$.

Continuando la disección llegamos a la bifurcación de la arteria mediana plantar en sus 2 ramas: profunda y superficial. La rama profunda con sus perforantes, da base al colgajo medialis pedis (Fig. 5). El diá-

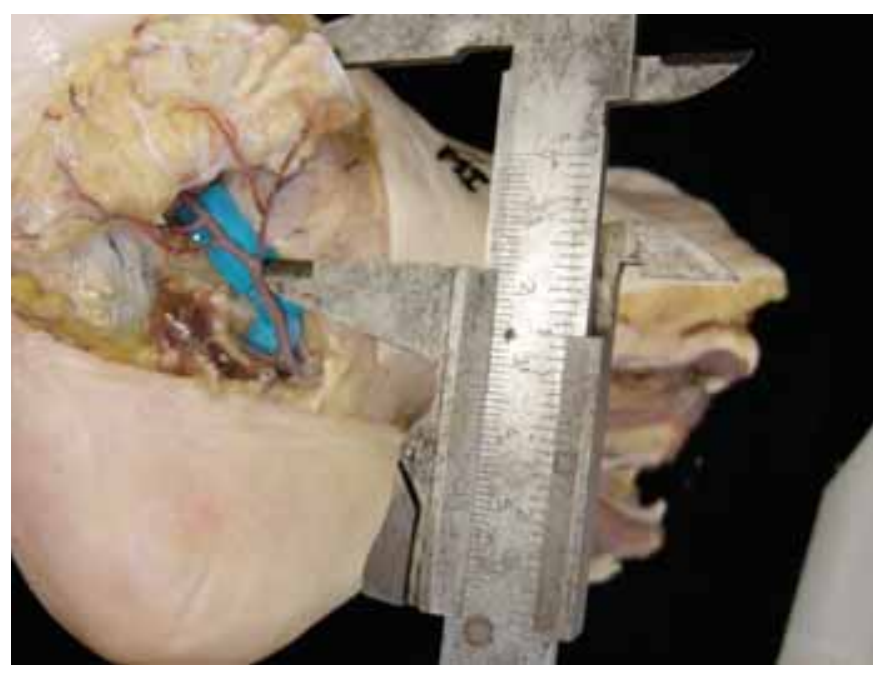

Fig. 6. Mediciones de los puntos de salida de las perforantes mediante tres puntos de referencia.

metro medio del pedículo de este colgajo es de 1,02 si lo medimos a nivel de la bifurcación y la longitud varía de 1,2 a $2,8 \mathrm{~cm}$.

En nuestro estudio llamamos zona III al área nutrida por perforantes de la rama profunda a partir de su bifurcación.

En cada perforante dominante realizamos las mismas mediciones hasta cada uno de los 3 puntos de referencia (Fig. 6). Finalmente, elaboramos una tabla (Tabla I) con mediciones de cada perforante en cuanto a su longitud

Tabla I. Mediciones del punto de salida de la perforante en relación con los 3 puntos de referencia, de la longitud y del diámetro de la perforante.

\begin{tabular}{|c|c|c|c|c|c|c|c|c|c|c|c|}
\hline & Caso 1 & Caso 2 & Caso 3 & Caso 4 & Caso 5 & Caso 6 & Caso 7 & Caso 8 & Caso 9 & Caso 10 & $\mathbf{M}$ \\
\hline \multicolumn{12}{|l|}{ P1 } \\
\hline MI & 3,8 & 4,8 & 3,8 & 3,8 & 4,2 & 4,1 & 3,4 & 5 & 4,9 & 3,8 & 4,16 \\
\hline ON & 3,1 & 2,3 & 3,2 & 3,7 & 3,6 & 3,8 & 2,7 & 3,5 & 4,5 & 3,2 & 3,36 \\
\hline M & 11,5 & 11,2 & 12 & 13,5 & 11 & 13,5 & 11,1 & 11 & 12 & 11,6 & 11,84 \\
\hline $\mathrm{L}$ & 1,1 & 1,5 & 2,3 & 1,5 & 1,3 & 2,6 & 1,1 & 1,8 & 1,6 & 1,9 & 1,5 \\
\hline $\mathrm{D}$ & 0,8 & 1 & 1,1 & 1,1 & 1 & 1,2 & 1 & 1 & 1,1 & 1,1 & 1,14 \\
\hline \multicolumn{12}{|l|}{ P2 } \\
\hline MI & 4,6 & 4,7 & 5,6 & 5,2 & 4,1 & 4,4 & 4,3 & 6,1 & 5,8 & 4,5 & 4,93 \\
\hline ON & 3,1 & 3,3 & 3,9 & 5 & 2,7 & 4,1 & 2,8 & 3,6 & 4,1 & 2,8 & 3,54 \\
\hline M & 10,2 & 12 & 12 & 12,6 & 9,8 & 11,8 & 11,3 & 10,9 & 11,1 & 10,5 & 11,22 \\
\hline $\mathrm{L}$ & 1,9 & 1,3 & 2,5 & 2,2 & 3 & 2,1 & 2,5 & 1,5 & 1,4 & 1,5 & 1,99 \\
\hline $\mathrm{D}$ & 0,8 & 0,7 & 1 & 1,1 & 1,1 & 1 & 0,8 & 0,8 & 0,8 & 0,8 & 0,89 \\
\hline \multicolumn{12}{|c|}{ MPD } \\
\hline MI & 7,4 & 8,2 & 7,8 & 7,3 & 5,5 & 8 & 8,1 & 7,7 & 7 & 6,5 & 7,35 \\
\hline $\mathrm{ON}$ & 3,6 & 3,3 & 3,4 & 3,8 & 1,5 & 3,4 & 3,2 & 3,2 & 3,8 & 3 & 3,22 \\
\hline M & 6,7 & 7,4 & 8,8 & 10,2 & 8,4 & 8,5 & 7,6 & 7,5 & 8,9 & 8 & 8,2 \\
\hline $\mathrm{L}$ & 2,2 & 1,2 & 2,2 & 2,8 & 1,5 & 2 & 1,3 & 2,4 & 1,6 & 2 & 1,91 \\
\hline $\mathrm{D}$ & 1 & 0,7 & 0,7 & 1 & 0,8 & 1 & 0,8 & 1 & 1 & 1,2 & 1,02 \\
\hline \multicolumn{12}{|l|}{ BF } \\
\hline MI & 3,8 & 4,3 & 4,4 & 5 & 4,5 & 4 & 4,2 & 4,6 & 5 & 4,5 & 4,43 \\
\hline ON & 5,1 & 3,8 & 5,2 & 5,4 & 4,5 & 5,1 & 4,1 & 5 & 5,1 & 4,3 & 4,76 \\
\hline M & 13,5 & 13,5 & 13,5 & 13,6 & 12,8 & 13,5 & 12,3 & 13,8 & 12,9 & 12,2 & 13,15 \\
\hline
\end{tabular}

P1 - perforante zona I, P2 - perforante zona II, P 3 - perforante zona III, MI - maleolo interno, ON - os navicularis, M - primera articulación metatarsiana, L - longitud de la perforante, $\mathrm{D}$ - diámetro de la perforante, $\mathrm{BF}$ - bifurcación de la medialis pedis a la rama profunda y superficial. 
desde su punto de salida hasta su punto de entrada en la piel y su sección transversal (Fig. 7).

\section{Discusión}

El pie siempre ha sido considerado como una zona difícil de reconstruir por su complejidad anatómica y por la imposibilidad de cierre directo, incluso en el caso de defectos de pequeño tamaño, debido a la falta de tejidos locales adecuados.

Las opciones quirúrgicas para la cobertura de defectos locales en el pie varían según el tamaño, la extensión y la localización de la lesión. En las lesiones menores de $3 \mathrm{~cm}$ se utilizan colgajos locales en las zonas de apoyo e injertos en el resto. En las lesiones mayores de $3 \mathrm{~cm}$ se aplican colgajos locales o colgajos libres fasciocutáneos, musculocutáneos o musculares mas injertos de piel. Si existe pérdida de tejido óseo se puede aplicar un colgajo libre osteocutáneo.

El colgajo de la planta del pie fue utilizado por primera vez por Mir y Mir para reconstrucción del pie contralateral mediante técnica de piernas cruzadas (4). Shanahan, en el año 1979, describió el colgajo mediano plantar que incluía el nervio mediano plantar; se utilizó para la cobertura del talón tras 1 semana de retardo (5). Este colgajo fue diseñado como colgajo con pedículo cutáneo y en el momento de rotarlo dejaba un importante exceso de piel. Harrison en el año 1981, publicó un caso de colgajo mediano plantar rotado sobre su pedículo vascular para cobertura del talón y confirmó la importancia de dividir el pedículo cutáneo dejando el colgajo basado sólo en el pedículo vascular (6).

El colgajo mediano plantar libre sensitivo fue descrito por primera vez por Morrison en 1983 y Sekiguchi lo describe como colgajo libre para la reconstrucción palmar en el año 1984 (7,8). En el año 1990 Masquelet publica un importante estudio anatómico sobre 30 cadáveres, en el cual describe la diferencia anatómica entre los colgajos mediano plantar y medialis pedis y presenta 5 casos de aplicación clínica del colgajo medialis pedis para la reconstrucción local del pie con pedículo proximal y con pedículo distal invertido (9).

En el año 1995, Zhang realizó un estudio anatómico sobre 33 cadáveres, en el que describe un colgajo de la rama cutánea de la arteria mediana plantar, con el propósito de disminuir la morbilidad de la zona donante, dejando intactos el tronco principal y la fascia plantar (10).

Morris, en 2006 presenta una detallada revisión sobre las técnicas disponibles para estudiar la anatomía vascular en cadáver (11).

En nuestro estudio presentamos un trabajo de disección realizado sobre 10 pies de cadáver en los

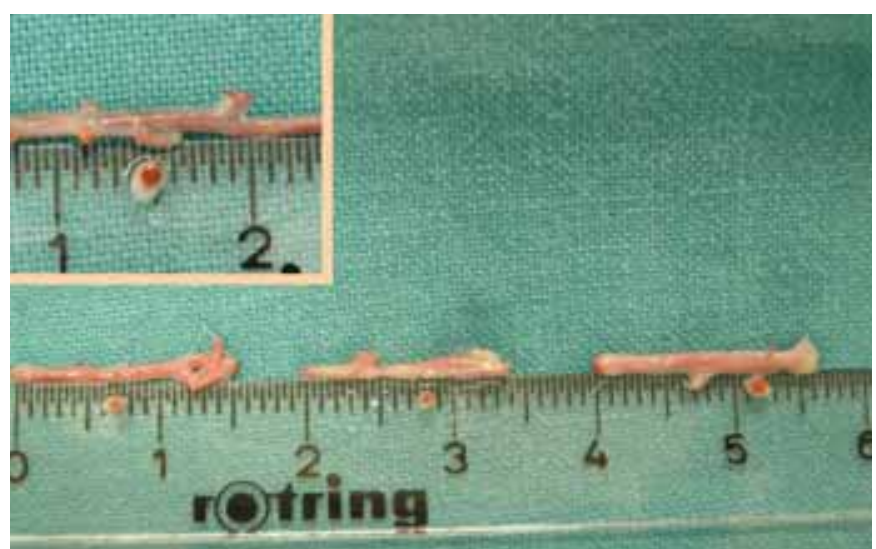

Fig. 7. Mediciones del diámetro y de la longitud de las perforantes.

que disecamos las perforantes dominantes de la arteria plantar medial. El diámetro medio y la longitud de cada perforante nos permiten utilizarlas como base para un colgajo local o para un colgajo libre.

La perforante I nutre una zona de grosor cutáneo muy fino que puede ser una buena indicación para reconstrucciones de la mano, sobre todo de los dedos.

El colgajo correspondiente a la perforante II fue descrito por Koshima como colgajo libre para reconstrucciones de los dedos de la mano (12) y como colgajo local para cobertura del talón y la zona metatarsiana (13). La piel de esta zona es de doble grosor que la de la zona I, por lo que el cierre directo de la misma es más complicado y normalmente la zona donante se cubre con un injerto de piel parcial. El grosor de este colgajo puede suponer una ventaja a la hora de reconstruir defectos del talón. Cuando queremos utilizar este colgajo como colgajo libre, en muchos casos deberemos incluir parte del tronco principal de la arteria mediana plantar en el pedículo, ya que en varias de nuestras disecciones, el diámetro de esta perforante era menor de $0,8 \mathrm{~mm}$.

El pedículo del colgajo medialis pedis que corresponde en nuestra disección a la zona III nutre un área bastante grande y permite levantar colgajos de $4 \times 9$ $\mathrm{cm}$, injertando de forma complementaria la zona donante. Wei, en el año 2001 describe una serie de casos de reconstrucción con este colgajo hecho en varios dedos de la mano simultáneamente (14).

$\mathrm{Al}$ utilizar este colgajo, para obtener un pedículo más largo y de mayor diámetro, se procede muchas veces a la ligadura de la rama superficial de la arteria plantar medial. Se puede llegar hasta la bifurcación de la arteria tibial posterior, pero en este caso se sacrifica el tronco principal de la arteria plantar medial. Otra opción posible, gracias a las comunicaciones de este vaso con el arco plantar, es el colgajo invertido para la cobertura de la zona metatarsiana, incluso con posibilidad de llegar a cubrir parte del dorso del pie (Fig. 8). Es un colgajo universal que puede ser perfectamente aplicado tanto para reconstrucciones locales como a distancia. 


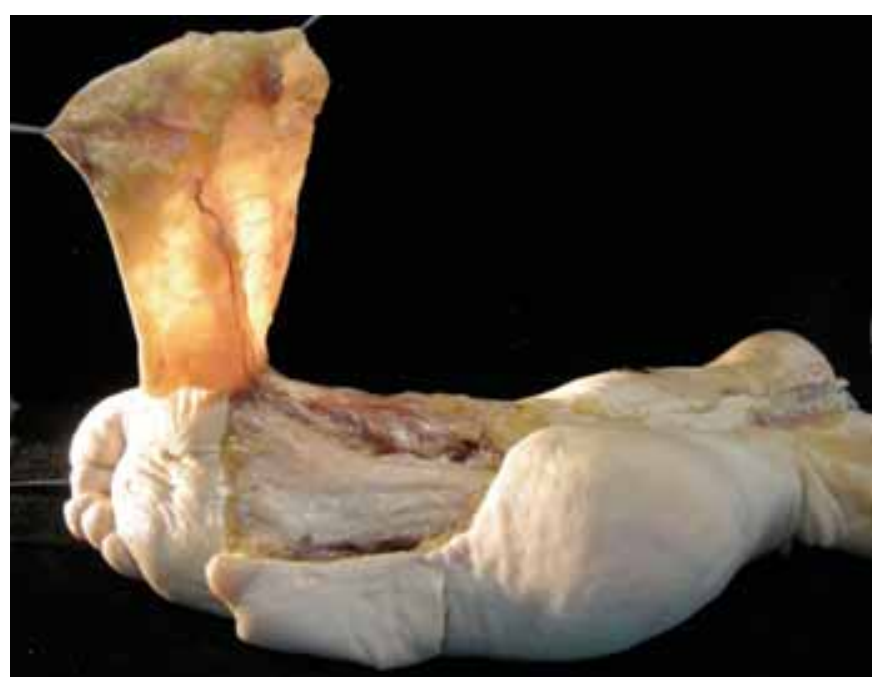

Fig. 8. Comunicaciones de la rama profunda con el arco plantar que dan base al colgajo invertido.

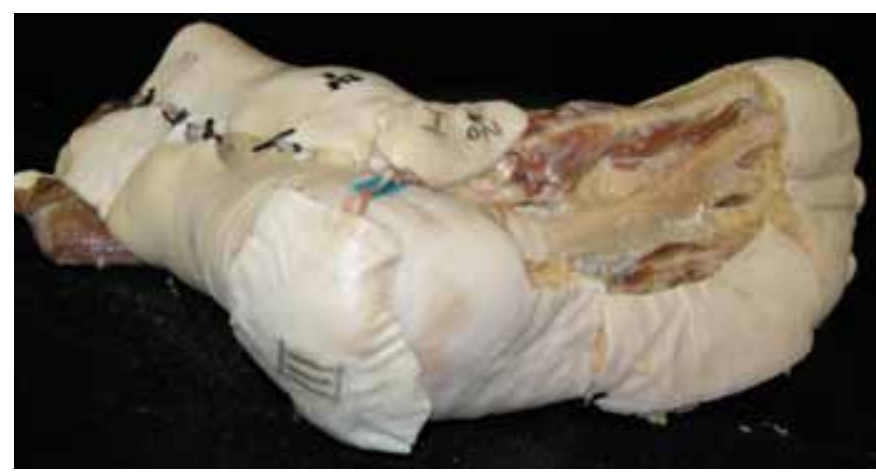

Fig. 9. Cobertura del talón con el colgajo medialis pedis.

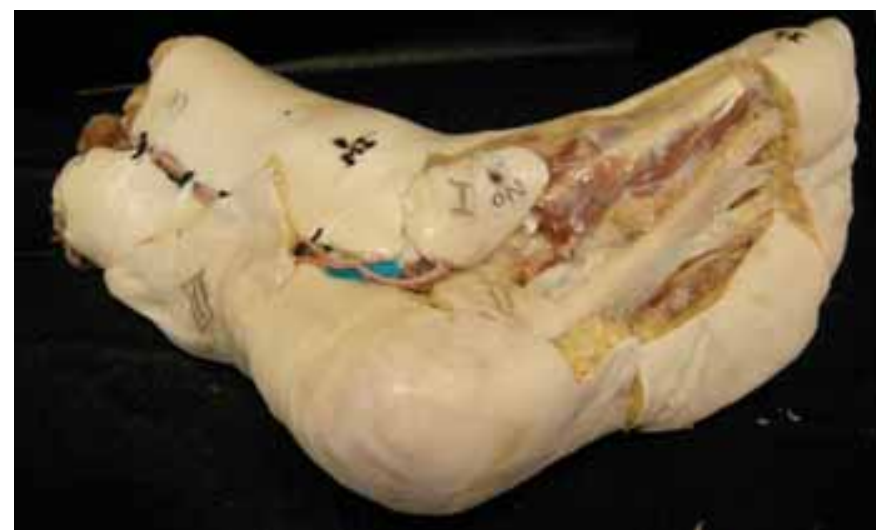

Fig. 10. Cobertura del tendón de Aquiles con el colgajo medialis pedis.

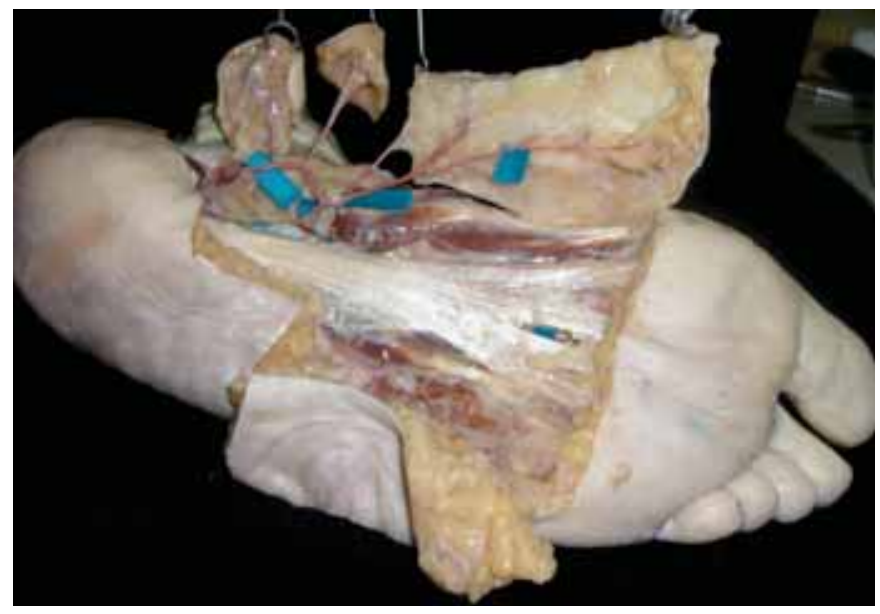

Fig. 11. Colgajos de las zonas I, II y III.
El pedículo de cada colgajo puede alargarse incluyendo la arteria mediana plantar, lo que se consigue continuando la disección hasta la bifurcación de la a. tibial posterior (Fig 9, 10).

Es evidente que la disección de los colgajos de las perforantes del píe es delicada y requiere una buena técnica microquirurgica. El diámetro de los vasos puede suponer un reto a la hora de realizar las anastomosis. Sin embargo, según varios casos clínicos publicados hasta hoy, estos colgajos tienen una buena supervivencia en manos de cirujanos experimentados.

A la hora de utilizar estos colgajos para reconstrucción de la mano tenemos la ventaja de evitar un daño adicional a la mano que ya ha sufrido un traumatismo.

En la reconstrucción local tenemos varias opciones (Fig 11) y podemos preservar el tronco principal de la arteria mediana plantar.

\section{Conclusiones}

Las principales zonas nutridas por las perforantes de la arteria mediana plantar pueden servir como base para colgajos diferentes, que pueden utilizarse tanto para reconstrucción local como para reconstrucción microquirúrgica a distancia.

Los colgajos de las zonas I y II pueden utilizarse para la cobertura de defectos pequeños. El colgajo de la zona III se aplica para la cobertura de defectos de mayor tamaño.

Estos colgajos están indicados sobre todo para reconstrucción del talón, del tendón de Aquiles o como colgajos libres para cobertura de defectos de la mano. También el colgajo de la zona III puede aplicarse en forma de colgajo invertido para la cobertura de la zona metatarsiana.

Los colgajos de los vasos perforantes del pie son una buena opción para la reconstrucción del pie y de la mano, con una mínima morbilidad de la zona donante y con un buen resultado funcional gracias a las características del tejido de esta zona.

\section{NOTA DEL AUTOR}

El trabajo de disección en cadáver en el Departamento de Anatomía Humana de la Universidad Miguel Hernandez de Elche, Alicante, fue realizado gracias a la Beca Crisvi ${ }^{\circledR}$ que fue entregada a la Dra. Prousskaia en concepto de premio, en el XLIII Congreso Nacional de la Sociedad Española de Cirugía Plástica, Reparadora y Estética (SECPRE), Zaragoza, España, 2008. 


\section{Dirección del autor}

Dr. Elena Prousskaia Peregudova

Servicio de Cirugía Plástica y Grandes Quemados. Hospital de Cruces

Plaza de Cruces s/n.

CP 48903, Baracaldo, Vizcaya. España.

e-mail: eprousskaia@hotmail.com

\section{Bibliografía}

1. Hansen JT, Lambert DR.: "Netter's Clinical Anatomy", First Edition, Elsevier Inc, USA, 2005, Chapter 4, Lower limb, Pp: 204-295.

2. Cormack GC, Lamberty BGH.: "The arterial anatomy of skin flaps", Second Edition, Longman Group Ltd, USA, 2004, Chapter The foot, Pp: 260-267.

3. Tank PW.: "Grant's dissector", 13th edition, Lippincott Williams and Wilkins, USA, 2005, Chapter 6, The lower limb, Pp 122-150.

4. Mir y Mir: "Functional graft of the heel". Plast. Reconstr. Surg, 1954, 14: 444.

5. Shanahan RE, Gingrass RP: "Medial plantar sensory flap for coverage of heel defects". Plast. Reconstr Surg, 1979, 64: 295.
6. Harrison DH, Morgan DGB: "The instep flap to resurface plantar defects". Br. J. Plast Surg, 1981, 3: 315.

7. Morrison WA, Grabb DM, O'Brien, BMCC, Jenkins A: "The instep of the foot as a fasciocutaneous island and as a free flap for heel defects". Plast Reconstr Surg, 1983, 72:56.

8. Sekiguchi J, Kajiyama K, Kobayashi S: "Application of the free medial plantar fasciocutaneous sensory flap to the hand". Journal of Japanese Society of the hand, 1984, 1: 705.

9. Masquelet AC, Romana MG: "The medialis pedis flap: a new fasciocutaneous flap". Plast. Reconstr. Surg, 1990, 85: 765.

10. Zhang GM, Ahmed SS, Tsai TM: "Anatomic study of a new axial skin flap based on the coetaneous branch of the medial plantar artery". Microsurgery, 1995, 16: 144.

11. Morris S, Tang M, Geddes R.: "Bases anatómicas vasculares de los colgajos perforantes cutáneos". Cir. plást. iberolatinoam. 2006, 32 (4): 225.

12. Koshima I, Katsuyuki U, Inagawa K, Hamasaki T, Moriguchi T: "Free medial plantar perforator flaps for the resurfacing of finger and foot defects". Plast. Reconstr. Surg. 2001, 107: 1753.

13. Koshima I, Mitsunaga N, Mihara M, Nakai I, Akazawa S, Fukuda N, Watanabe Y, Nakagawa M: "Island medial plantar artery perforator flap for reconstruction of plantar defects". Ann. Plast. Surg, 2007, 59: 558.

14. Tsai FC, Cheng MH, Chen HC, Wei FC: "Microsurgical medialis pedis flaps for reconstruction of soft-tissue defects in the hand". Ann. Plast. Surg, 2001, 48: 41. 